\title{
Enhancement and Design Considerations Distributed FACTS for Mitigation of Power Quality problem
}

\author{
${ }^{1}$ Madhuri Uparwat, ${ }^{2}$ D. B. Meshram, ${ }^{3}$ S. Dutt \\ ${ }^{I}$ M.Tech (Energy System*)Department Of Electrical Engineering, RCERT-Chandrapur (MS), Indian \\ ${ }^{2}$ Asso. Professor Department Of Electrical Engineering, RCERT-Chandrapur (MS), Indian \\ ${ }^{3}$ Asso. Professor Department Of Electrical Engineering, RCERT-Chandrapur (MS), Indian
}

\begin{abstract}
This paper introduces the concept of a distributed static series compensator (DSSC) for Flexible AC transmission systems (FACTS) devices can control power flow in the transmission system to improve asset utilization, relieve congestion, and limit loop flows. High costs and reliability concerns have restricted their use in these applications. The concept of distributed FACTS (D-FACTS) is introduced as a way to remove these barriers. A new device, the distributed static series compensator (DSSC), attaches directly to existing HV or $E H V$ conductors and so does not require $H V$ insulation. It can be manufactured at low cost from conventional industrial-grade components. The DSSC modules are distributed, a few per conductor mile, to achieve the desired power flow control functionality by effectively changing the line reactance. Experimental results from a prototype module are presented, along with examples of the benefits deriving from a system of DSSC devices.

Index Terms: Distributed FACTS, Flexible AC Transmission Systems (FACTS), interconnected power systems, MATLAB Simulink and programming, Power flow control.
\end{abstract}

\section{Introduction}

The power grid in the India and in most other parts of the world, is aging and under increasing stress. The modern industrial infrastructure demands increasing amounts of affordable and reliable electricity. Yet in a semi-regulated utility environment and in the face of increasing public sentiment against locating power lines in their communities, the ability to use the existing asset base more effectively has become a critical issue [1].

The utilities have done a good job in ensuring availability of reliable electricity. An important component of higher reliability is a gradual move from radial power distribution to a system that is increasingly networked. This allows faulted sections of the grid to be rapidly isolated, without sustained interruption of power to the vast majority of customers. The utility cannot effectively control how power flows on such a network. Further, the first line that hits thermal limit constrains the total power transfer capacity of the entire system, even as other lines in the system are only operating at a fraction of their capacity. Finally, the network topology changes continually, as lines, loads and generation are added and dropped. Maintaining system integrity under current Conditions are very important aspects. The 'conventional' and technically proven approach for controlling power flow on the grid has been through the use of Flexible AC Transmission Systems (DFACTS) devices [2].

The concept of DSSC is on the base of utilizing a low power single-phase inverter, which attaches to the transmission conductor and dynamically controls the corresponding transfer impedance. By this way, the active control of power flow on the line is achieved [1]. Quite few papers have attempted the modeling and interrogating of DSSC's capabilities. For instance, [1] offers a graphical simulation model for DSSC and explores a single phase system which comprises only one DSSC and an ideal voltage source instead of generators. Hence, the least available and reported technical papers for DSSC justifies further studies on the other capabilities of this device. This study serves a research where 1400 DSSCs are integrating in a two-area, two-machine system in order to examine the transient stability of the system. With the aim of improving the transient stability, a supplementary controller has been designed and suitably combined to the main control loop of DSSCs. Simulation results exhibit the efficient influence of DSSCs in the transient stability augmentation and justifies its controller performance.

\section{The Distributed Static Series Compensator (DSSC)}

A controlled transmission system can be made up of a large number, e.g., hundreds or thousands, of DSSC modules, each module containing a small rated (1-20 kW) single phase inverter, a communications link and a single turn transformer (STT) that is mechanically clamped on to - and suspended from-the transmission line conductor (or insulator). The STT uses the transmission conductor as a secondary winding, directly injecting the desired voltage into the cable itself. The inverter is self-powered by induction from the line, and can be controlled to inject a voltage that is orthogonal to the line current directly into the conductor. The module can either be suspended from the conductor or configured as a replacement for the conductor support clamp on 
an insulator. Further, since it does not require supporting phase-ground insulation, the module can easily be applied at any transmission voltage level. Figure 2 shows an electro-mechanical concept diagram for a typical DSSC module, while Fig. 3 shows the power circuit schematic. The mechanical form of the module may either clip-on to the conductor, as shown in Fig. 2, or may be incorporated into the insulator suspension clamp, avoiding any concern about weight and conductor vibration damage.

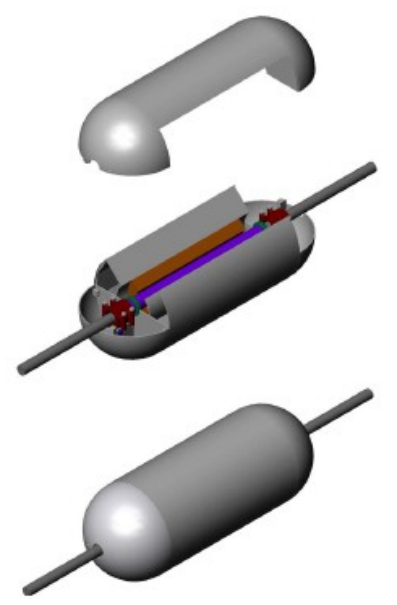

Fig: DSSC concept showing clam on capability

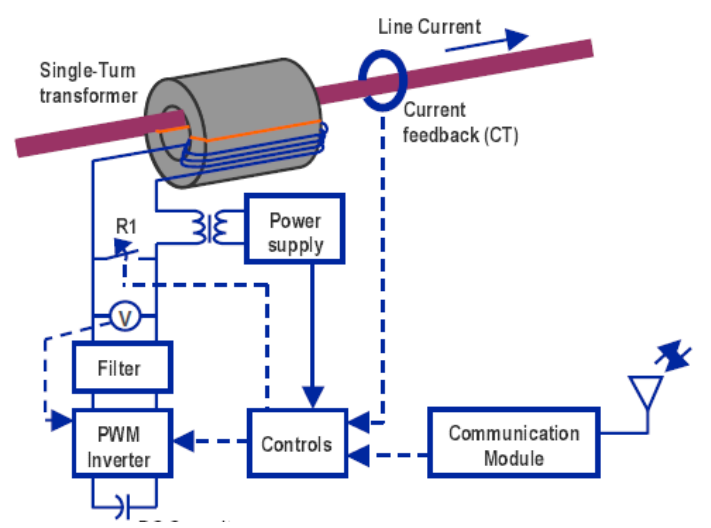

Fig: DSSC circuit

When the transmission line is not powered up, the STT is bypassed by a normally closed relay contact (R1) that opens once control power is available. A current transformer is used to generate control power, allowing the DSSC module to operate as long as the line current is greater than a minimum level, say $150 \mathrm{~A}$. The line appears to the inverter as an inductive current source. The single phase inverter uses four IGBT devices along with an output LC filter and a dc bus capacitance. The inverter output voltage is controlled using pulse width modulation techniques, and has two components. The first is in quadrature with the line current, and represents the desired impedance to be injected. The second is in phase with the line current, and allows compensation of power losses in the inverter, and regulation of the dc bus of the inverter. System commands for gradual changes are received from a central control center using a wireless or power line communication (PLC) technique [8]. In the event of rapid transients or faults the DSSC modules can be programmed to operate autonomously. With the DSSC attached and operating at the conductor potential, conductor temperature measurement capability is easily added and actual temperature readings can be communicated to the central system controller.

The STT is a key component of the DSSC module. It is designed such that the module can be clamped onto an existing transmission line. The STT is designed with a high turns ratio, say 75:1. This implies that under a normal line current of say $1500 \mathrm{~A}$, the inverter would only handle 20 amperes. Designing the inverter for 500 volts rms output would then allow the DSSC module to inject $7 \mathrm{~V}$ rms leading or lagging, corresponding to \pm 10 $\mathrm{kVAr}$ in series with the line under normal operating conditions. It is anticipated that such a module could be designed to weigh less than $45 \mathrm{~kg}(100 \mathrm{lb})$, making the module suitable for direct clamp-on mounting on the transmission conductor.

The STT also allows the inverter to possibly continue operating under fault conditions. For instance, at a fault current of 50,000 A, the inverter current is still only $667 \mathrm{~A}$, well within the capability of commercially 
used IGBT devices. This raises the interesting possibility that unlike TCSC's, the DSSC could regulate line impedance under normal conditions, switching into a maximum inductance injection mode within microseconds to prevent an increase in the fault current levels. The inverter ratings clearly demonstrate that the semiconductors and components used are commercially available in very high volumes for the motor drives, UPS, and automotive industries, thus validating the potential for realizing low cost.

\section{Dssc Impact On Power Flow}

As mentioned earlier, DSSC is connected in series to the transmission line and thus has the ability of injecting a Synchronous fundamental voltage that is in quadrature with the line current directly into the transmission conductor. As a result, the transmitted power becomes a parametric function of the injected voltage and can be stated as the sequel:

Where:

$$
P_{12}=\frac{V_{1} V_{2}}{X_{L}} \sin \partial \frac{V_{1} V_{q}}{X_{L}} \cos \frac{\partial}{2}\left[\frac{\sin \frac{\partial}{2}}{\left(\frac{V_{1}+V_{2}{ }^{2}}{2 v_{2}}\right)} \frac{V_{1}}{V_{2}} \cos ^{2} \frac{\partial}{2}\right]
$$

$\mathrm{V} 1$ and $\mathrm{V} 2=$ the bus voltage magnitudes;

$\mathrm{Vq}=$ the series injected voltage magnitude;

delta $=$ the voltage phase difference and $\mathrm{XL}=$ the impedance of the line, assumed to be purely inductive.

The DSSC can simply increase the transmittable power as well as decrease it by reversing the polarity of the injected ac voltage [15]. This is worth noting that this feature is responsible for corroborating the DSSC salient ability for power flow control in the overall system. The variation of the transmitted power verses load angle with different quadrate voltage injections, for equal bus voltage magnitudes is depicted in Fig. 3.

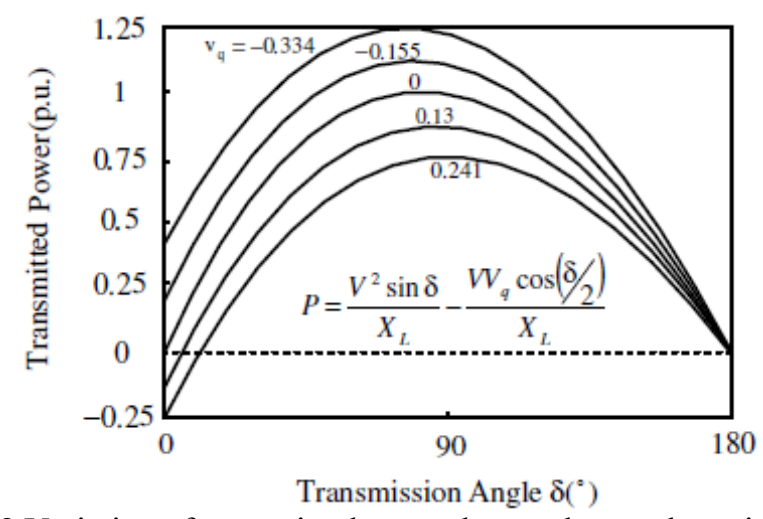

Fig. 3 Variation of transmitted power by quadrate voltage injection

\section{Simulation Model Extracted For Dssc}

This section reviews the graphical-based simulation model for the DSSC introduced in [11]. This model provides comprehensive understanding of the operational principles of the DSSC; and hence can be very appropriate for extra operational analysis of this device.

\section{A. Single Phase Inverter Structure}

As displayed in Fig. 4, the DSSC power circuit includes the inverter, filter circuit, breaker, and transformer. As shown, the DSSC single phase inverter consists of four IGBT devices in a full bridge configuration. The dc link is realized with a fixed capacitor. Also an output LC filter ( $\mathrm{Lf}$ and $\mathrm{Cf}$ ) is expected in the output of the inverter to alleviate the harmonic pollution of the injected voltage. 


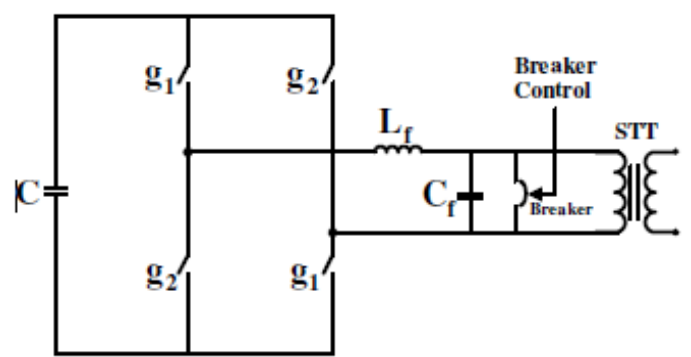

Fig. 3 DSSC power circuit

Sinusoidal pulse width modulation (SPWM) technique is well known to offer simplicity and good response for inverter switching strategy. On account of this reason, SPWM is the case which is speculated here.

\section{B. Control Strategy}

The fundamental task of the DSSC is to control the power flow in a transmission line. This goal can be obtained either by direct control in which both the angular position and the magnitude of the output voltage are controlled, or by indirect control in which only the angular position of the output voltage is to be controlled and the magnitude remains proportional to the de terminal voltage [16].

The inverters which are directly controlled impose more difficulty and higher cost to be implemented compared to indirectly controlled inverters, also their function is typically correlated with some penalty in terms of increased losses, greater circuit complexity and increased harmonic components in the output. As a consequence, the control scheme used for the DSSC model investigated in this paper is based on indirect control technique [11].

Fig. 5 exhibits the DSSC control system and SPWM generator. The controller main objective is to hold the charge constant on the dc capacitor and also to inject a voltage that is in quadrature with the line current. A small phase displacement namely, error, beyond the required $90^{\circ}$ between the injected voltage and the line current is needed to fix the dc capacitor voltage. The signal obtained by comparing Vdc with Vdc(ref) is passed through a proportional integral (PI) controller which generates the required phase angle displacement or error. The Phase-Locked Loop (PLL) provides the basic synchronization signal, $\theta$ which is the phase angle of the line current [11].

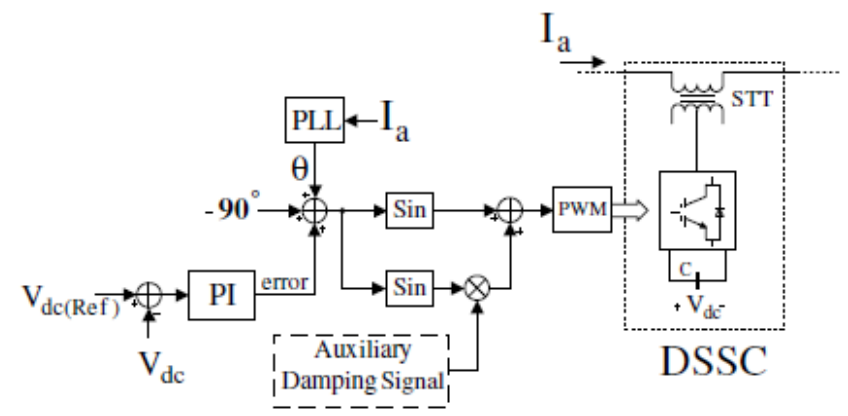

Fig. 5 DSSC control system and SPWM generator

\section{Transient Stability Enhancement With Dssc}

DSSC would enhance the transient stability by partial eliminating of the series impedance of the transmission line. The transient stability however, can be more increased by temporarily changing the compensation with a supplementary controller combined to the main control loop of DSSCs. For the duration of the first acceleration period of the machine, the controller increases the transmitted power by injecting higher series voltage. Similarly, the deceleration of the machine is increased simply by increasing the line impedance and thus, decreasing the transmitted power. Fig. 6 shows the power system considered as the case study in the following simulations with respect to Fig. 6, it can be observed that the load center is modeled by a $5000 \mathrm{MW}$ resistive load. The load is fed by a local generation of $4000 \mathrm{MW}$ (machine G2) and a remote $1000 \mathrm{MW}$ plant (machine G1) which is connected to the load center through a long $500 \mathrm{kV}, 700 \mathrm{~km}$ transmission line. The system has been initialized so that the line transmits $950 \mathrm{MW}$ which is close to its surge impedance loading (SIL=977 MW)

$\mathrm{Vdc}$ (ref) for each DSSC module is fixed at $2 \mathrm{kV}$, amplitude modulation ratio is set at 0.5 , and the turns ratio of STT is 1:100. Consequently, by applying these adjustments, the injected voltage of each DSSC module is anticipated to reach a peak to peak value of $10 \mathrm{~V}$. Regarding that the injected voltage of each DSSC is $10 \mathrm{~V}$, 
with the view of achieving $\% 4$ compensation on transmission line, near 1400 DSSC modules are required in each phase of the line. Fig. 6 provides a good illustration of DSSCs placement in the transmission lines.

$$
\begin{aligned}
& \qquad X_{L}=230 \Omega X_{i n j}=-9.5 \Omega \\
& \frac{X_{i n j}}{X_{L}} \times 100 \% \text { Companssion }
\end{aligned}
$$

The negative sign for inj $\mathrm{X}$ denotes the capacitive mode of DSSCs to series compensation of the line. In order to evaluate the DSSC impact in the transient stability enhancement, three different case studies are considered. The next section would present the complete simulation results for these states.

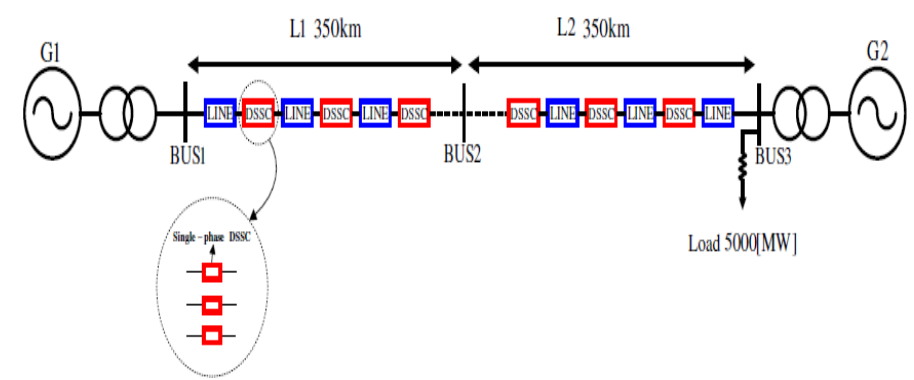

Fig. 6 Simulation model of two-machine power system for transient stability study with DSSCs

\section{Simulation Result}

This section is dedicated to scrutinize the DSSC influence in the overall system performance under three different cases. The cases considered here are as follows.

- Impact of DSSC on steady state operation point;

- Three phase fault - impact of DSSC without damping controller; and

- Three phase fault - impact of DSSC equipped with damping controller.

In the subsequent sections, simulation results are obtained for each situation individually and a complete discussion is presented.

\section{A. Impact of DSSC on Steady State Operation Point}

First of all, 1400 DSSCs are considered in the line per phase for achieving \%4 compensation. Fig.7 demonstrates that when the DSSCs are out of service, the rotor angle difference, d_theta1_2, between the two machines is about 53 degree.

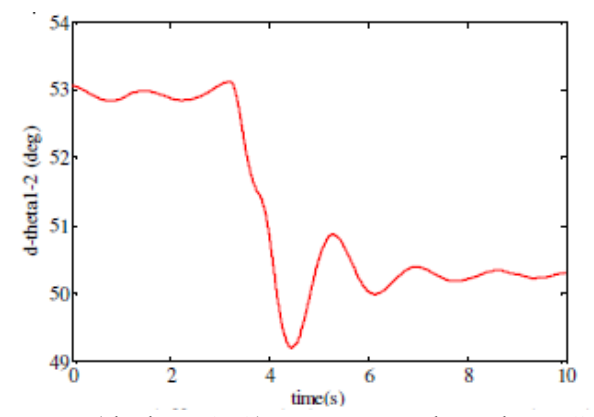

Fig. 7 The rotor angle difference (d_theta1_2) response when the DSSCs are put in service at $t=4$

Typically the line power is assumed to be constant; thus by entering the DSSCs to the power system at $\mathrm{t}=4 \mathrm{sec}$, the series impedance of the line will decrease. As a result, with respect to (3), the rotor angle difference d_theta1_2 is decreased to 50.25 degree. For this reason the transient stability margin of the system is improved with compensation.

$$
P_{12}=\frac{V_{1} V_{2}}{X_{L}} \operatorname{Sin} \delta
$$




\section{B. Three Phase Fault Impact of DSSC without Damping Controller}

Here, the DSSCs are initially placed in the circuit, but there is no damping controller on the main control loop, namely Auxiliary Damping Signal is set to zero. The bus1 near the machine G1 is subjected to a three phase to ground fault with duration of 0.085 second.

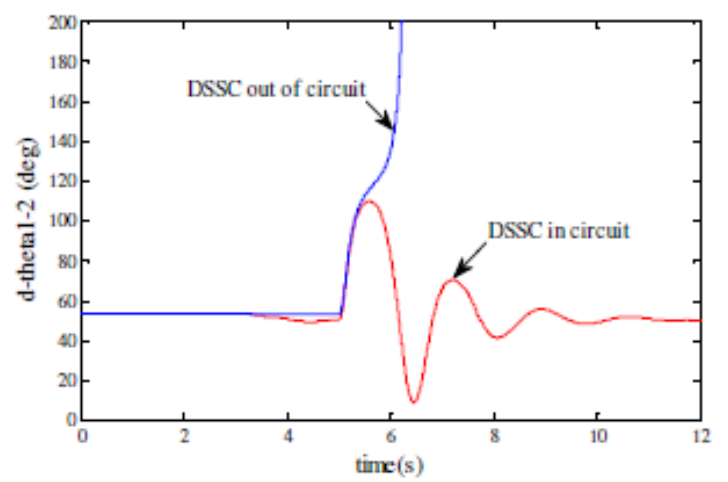

Fig: The rotor angle difference (d_theta1_2) variation after the fault without DSSCs and with DSSCs (without damping controller).

Figs. 8 and 9 are obtained for this case. It can be seen that, when the DSSCs are out of service, the rotor angle between the machines is increased rapidly and two machines fall out of synchronism after fault clearing. In contrast, when the DSSCs are in circuit, for the same fault circumstance the system remains stable.

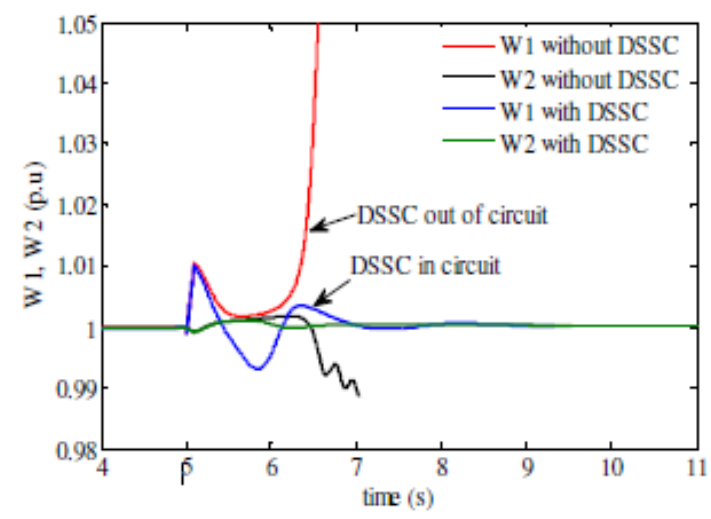

Fig: variation of the machines angular speed after the fault without DSSCs and with DSSCs (without damping controller)

\section{Three Phase Fault_Impact Of DSSC With Damping Controller}

To be more precise, the DSSC by itself does not provide the essential damping of oscillations as its primary duty is to control the line power flow. With the purpose of achieving better damping over a wide range of operation, a power oscillation damping (POD) controller is added to the main control loop of DSSCs. Fig. 10 shows the POD controller structure.

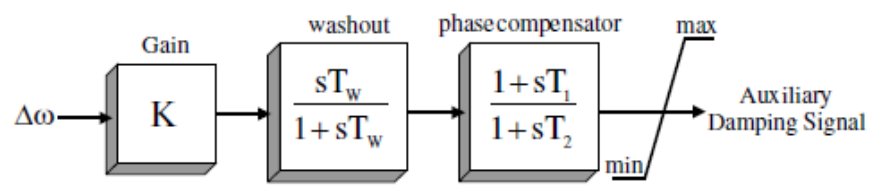

Fig: 10 POD controller structure.

This figure displays that the damping controller is composed of a gain block, a washout filter, and a lead-lag compensator. The damping controller is designed so as to provide an extra electrical torque in phase with the speed deviation in order to enhance the damping of oscillations [1]. The gain setting of the damping controller is adopted so as to achieve the desired damping ratio of the electromechanical fluctuations. The purpose of the washout circuit is to block the auxiliary controller from responding to the steady-state power 
conditions. The parameters of the lead-lag compensator are adjusted so that the phase shift between the speed deviation and the resulting electrical torque at the desired frequency is compensated. In the following, an additional electrical damping torque output is acquired in phase with the speed deviation. Here, the parameters of the controller are determined through the simulation studies by a trial-error method with the aim of achieving the best damping. The selection of an appropriate input signal is a fundamental issue in the design of an effective and robust auxiliary damping controller. In this paper, as depicted in Fig. 10, the generator rotor speed is considered as the input signal.

The output of the auxiliary damping controller is used to modulate the reference setting of DSSC in order to provide the excellent damping [16]. For the work at hand, as illustrated earlier in Fig. 5, the output of the POD controller is utilized to regulate the magnitude of the series injected voltage during electromechanical transients to yield the proper damping of oscillations. Now the system is subjected to a severe fault with duration of $0.1 \mathrm{sec}$ which is applied again near the bus1. Simulation results are presented in Figs. 11 and 12. These figures address two different cases namely, DSSC without any supplementary damping controller and DSSC which is supplied with POD controller. As it can be seen from Figs. 11 and 12, for the case where the DSSC lacks a power fluctuations damping controller, the system is completely unstable and two machines fall out of synchronism quickly. Also it can be noticed that when the DSSC control loop includes a power oscillation mitigating controller, the system is kept stable. Fig. 13 shows the POD controller output signal generated for damping the system oscillations

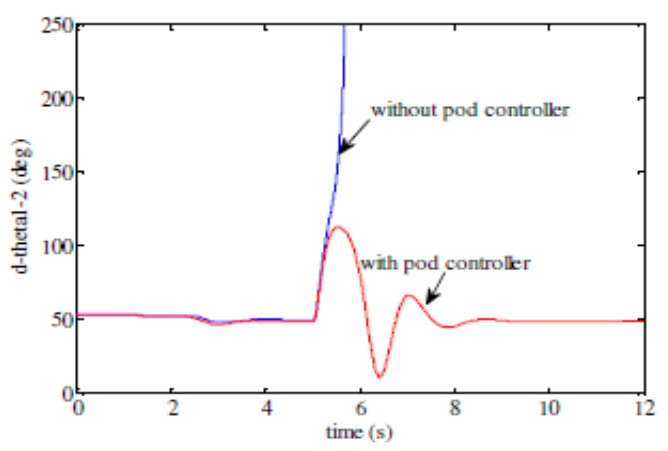

(a)

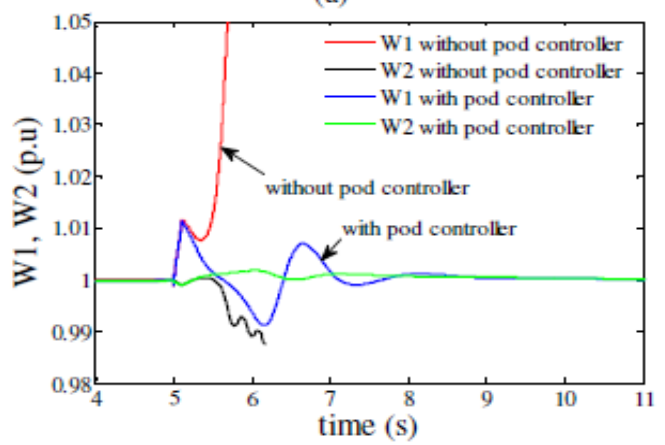

Fig: (a) the rotor angle difference (d_theta1_2) variation (b) variation of the machines angular speed after the fault with POD controller and without POD controller.

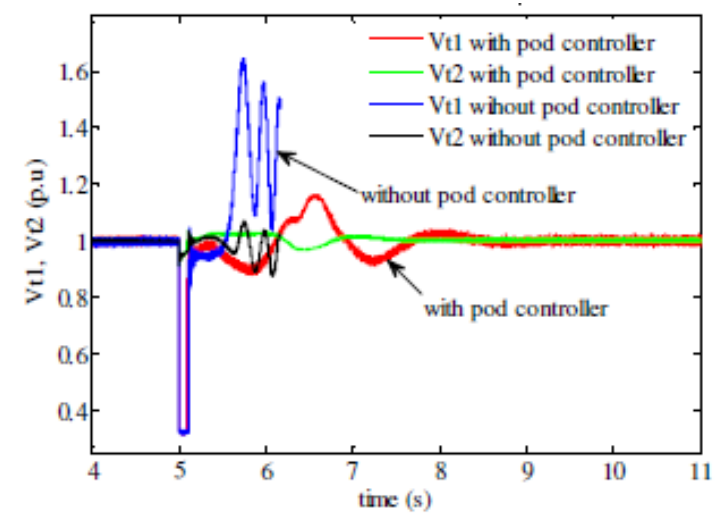

Fig. 12 Machines voltage variation after the fault with POD controller and without POD controller 


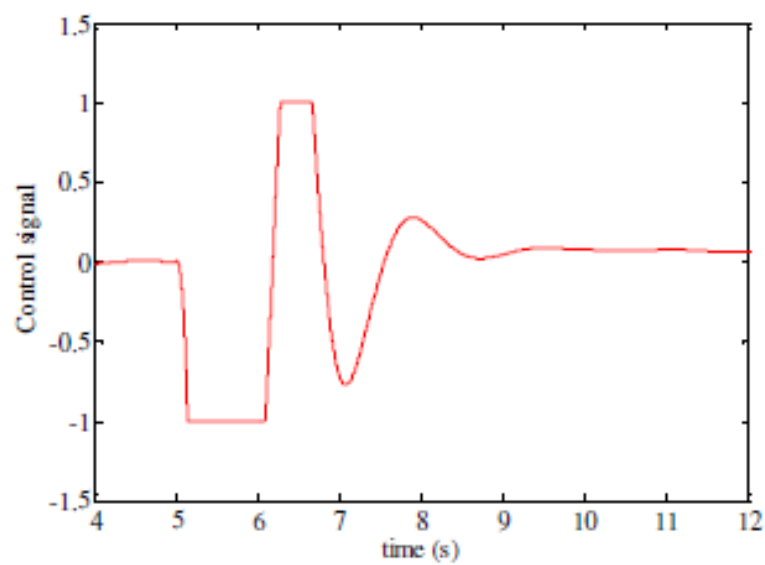

Fig. 13 Auxiliary Damping Signal generated with POD controller

\section{Conclusion}

This paper has presented the novel concept of a distributed static series compensator (DSSC).The development of D-FACTS devices has been announced as an effective approach to overwhelm the high cost implementation of FACTS family. The distributed devices are also apt to accomplish some other ancillary duties such as transient stability enhancement, power oscillation damping, etc. This study served a review of graphicalbased simulation model for the DSSC which is in fact a smaller counterpart of SSSC. A two-machine power system is put under investigation in order to verify the DSSC capability for increasing the transient stability of the whole system. Simulation results demonstrate that when the DSSCs are out of service, the rotor angle between the machines, d_theta1_2, is increased rapidly and two machines fall out of synchronism after fault clearing. But when the DSSCs are in circuit, they stabilize the system even without a specific controller. In the next, a severe fault is taken to occur in the system. It is shown that for this case, the system even with DSSCs in service becomes totally unstable. Hence, a POD controller is added to the main control loop of DSSC for improving the transient stability margin of the system. Simulation results exhibit that in this case the system will remain stable after the fault removal. Consequently, the ability of distributed devices such as DSSC in the enhancement of the power system operation is certified

\section{Acknowledgment}

The authors gratefully acknowledge the contributions of his work on the Enhancing DSSC aspect and mitigate the problem of power quality

\section{References}

[1]. P. Kundur, Power system stability and control, Prentice- Hall,N. Y, U. S. A, 1994

[2]. N. Hingorani, "Flexible AC Transmission,” IEEE Spectrum, v. 30, No. 4, pp. 40-45, Apr. 1993.

[3]. D.J. Gotham and G.T. Heydt, "Power Flow Control and Power Flow Studies for Systems with FACTS Devices," IEEE Trans. Power Systems, Vol. 13, No. 1, Feb. 1998.

[4]. M. S. El-Moursi, A. M. Sharaf, and K. El-Arroudi, "Optimal control schemes for SSSC for dynamic series compensation," Elect. Power Syst. Res., vol. 78, no. 4, pp. 646-656, 2008

[5]. L.J., Cai and I., Erlich. "Simultaneous coordinated tuning of PSS and FACTS damping controllers in large power systems", IEEE Trans. Power Syst, vol. 20, No. 1, pp. 294- 300, 2005.

[6]. S. Abraham, National Transmission Grid Study, U.S. Dept. of Energy, May 2002. Accessed April 2004 at http://www.eh.doe.gov/ntgs/.

[7]. W. J. Museler, President and CEO of the New York Independent System Operator (NYISO), presentation May 22, 2003,

[8]. http://www.nyiso.com/topics/articles/news_releases/2003/pa3_presentation.pdf

[9]. www.mathworks.com

[10]. www.ieee.com. 